\title{
Computing a Predictor Set Influence Zone through a Multi-Layer Genetic Network to Explore the Role of Estrogen in Breast Cancer
}

\author{
Leandro de A. Lima ${ }^{1,2}$, Marcelo Ris ${ }^{1}$, Junior Barrera ${ }^{3}$, Maria M. Brentani ${ }^{2,4}$, Helena Brentani ${ }^{4 *}$ \\ ${ }^{1}$ Instituto de Matemática e Estatística, Universidade de São Paulo, São Paulo, Brazil \\ ${ }^{2}$ Hospital A.C. Camargo, São Paulo, Brazil \\ ${ }^{3}$ Departamento de Física e Matemática da FFCLRP, Universidade de São Paulo, Ribeirão Preto, Brazil \\ ${ }^{4}$ Faculdade de Medicina, Universidade de São Paulo, São Paulo, Brazil \\ Email: "helena.brentani@gmail.com
}

Received July 5, 2012; revised August 10, 2012; accepted August 20, 2012

\begin{abstract}
Modeling inter-relationships of genes over a specific genetic network is one of the most challenging studies in systems biology. Among the families of models proposed one commonly used is the discrete stochastic, based on conditionally independent Markov chains. In practice, this model is estimated from time sequential sampling, usually obtained by microarray experiments. In order to improve the accuracy of the estimation method, we can use biological knowledge. In this paper, we decided to apply this idea to study the role of estrogen in breast cancer proliferation. The $n$-influence zone of a set $S$ of genes in a given multi-layer genetic network is a set $L$ of genes regulated, directly or indirectly, by genes in $S$, after at most $n$-1 layers. In this manuscript we describe a new approach for computing the $n$-influence zone of $S$ through the estimation of a multi-layer genetic network from gene expression time series, measured by microarrays, and biological knowledge. Using seed genes related to cell proliferation, our method was able to add to the third layer of the network other genes related to this biological function and validated in the literature. Using a set of genes directly influenced by estrogen, we could find a new role for cell adhesion genes estrogen dependent. Our pipeline is user-friendly and does not have high system requirements. We believe this paper could contribute to improve the data mining for biologists in microarray time series.
\end{abstract}

Keywords: Genetic Regulatory Networks; Estrogen; Time-Course Microarrays

\section{Introduction}

Genes are translated into proteins, which in turn can react to create complexes that regulate genes. This feedback process generates dynamical systems, known as genetic regulatory networks (GRN) that regulate metabolic pathways. In general, GRNs are very complex due to the intrinsic nonlinearity of the phenomena and the huge amount of variables (e.g. genes and proteins) involved.

A requirement for understanding quantitatively this natural phenomenon is the capacity of measuring it. In order to do that, we can use microarray [1] or RNA-Seq [2], which are technologies that permit to measure simultaneously the expressions of thousands of genes. This technology can be used to get instantaneously the state of nature under the experimental conditions defined by scientists. Thus, using a large experimental preparation and extracting relatively small volumes periodically, it is possible to measure gene expression profiles that are ${ }^{*}$ Corresponding author. samples of the dynamical behavior of genes. The resulting data is the source for the explosion of molecular profiling studies and permit the understanding of regulation mechanisms and, consequently, of biological phenomena associated to a specific organism or a cell culture [3]. When the study needs the measurement of expression profiles for a period of time, the time-course microarray experiment usually is the option. The analysis of these data permits to cluster genes sharing similar temporal profiles [4] and to estimate the architecture of GRNs [5].

There are several studies trying to model and estimate GRNs. A review of them can be found in [6-9]. The architecture of a GRN indicates the dependence of a gene dynamics to other genes dynamics. The model parameters can be estimated from promoter region structure analysis, gene expression profiles and biological knowledge. However, investigating large networks is very hard due to the small samples of the dynamical behavior of network genes (i.e. short gene expression profile) available. Studying specific gene networks is a more tractable 
problem [10]. The first model adopted to represent GRNs were Boolean state machines [11], also called Boolean Networks. Probabilistic Boolean Networks (PBNs) [12] are an extension of Boolean Networks in which the Boolean function to determine the next state in the network is not deterministic, being chosen each iteration from a family of Boolean functions according to a given probability distribution. Probabilistic Genetic Networks (PGNs) [13] is another mathematical view of PBNs that focus only on the probability distributions that characterize the PBNs. In fact, a PGN is a discrete Markov chain, whose states are vectors of gene expression, which obey some axioms: 1) the transition function is time-independent, i.e. the probability of a state, given a previous one, does not vary in time; 2) all the transition probabilities are positive, i.e. all the states given a previous one can occur; 3 ) the transition function is conditionally independent; 4). the transition function is almost deterministic, i.e. there is a state almost determined given a previous one. These axioms are motivated by a compromise between biological phenomena representation and difficulties with estimation from the available data.

In fact, any chosen model needs to be estimated from the available data, which is usually small. This is an important constraint in the choice of models, since complex models with many parameters would be impossible to estimate from reduced data sets. In these conditions, PBNs or PGNs are good options.

In this work, we used PGN to model GRNs and proposed a new algorithm to estimate them. The input data for this algorithm is a time-course microarray experiment, a subgroup of initial genes (the initial set of predictors, also called seed genes) and some prior knowledge of genes involved with the studied phenomena, whereas the output is a graph representing the architecture of the network designed. The genes that appear in the network are the influence zone of the seed genes (i.e. the genes influenced by the seed genes). Several manners of estimating GRNs have been proposed. Some of them propose, instead of computing the relationship between every pair of genes, to grow the network around specific genes $[5,14]$. And some propose to study the networks in specific contexts $[10,15]$. In this paper, we modified some aspects of the model for network estimation from seed genes: 1) designing the network through a sequential multi-layer estimation; 2) measuring the prediction capacity by the estimated mean conditional entropy; 3 ) proposing a formal model for using categorical biological knowledge to diminish the prediction estimation errors. Besides, the results were tested in microarray time series for studying genes regulated by estrogen.

Estrogen has a fundamental importance in the reproductive tissues [16] - the growing of mammary glands and endometry during pregnancy are estrogen-dependent
[17] — and it can also be related to the growing of tumor cells. There are more than 300 known genes with regulation positive or negative by estrogen [18-23]. These genes can be classified in categories by their bio- logical functions. Genes addressed to biological func- tions associated to cell proliferation are related to cancer [24] and estrogen can up-regulate or down-regulate those genes [19]. Taking it into account, we chose a dataset related to estrogen to test our method. Two examples of application will be shown. The input data is a timecourse microarray experiment of estrogen response in $T 47-D$ cells [22] treated with estrogen (E2) during 24 hours. In both experiments, a subgroup of genes (seed genes) regulated by estrogen was selected to start the network. The output is a graph representing interactions among genes and their predictors. The genes in the estimated network are the influence zone of the seed genes and their biological functions are analyzed in the context of the seed genes biological function

\section{Methods}

\subsection{Overview}

The $n$-influence zone of a set $S$ of genes in a given multi-layer genetic network is a set $L$ of genes regulated, directly or indirectly, by genes in $S$, after at most $n-1$ layers. We present an approach for computing the $n$-influence zone of the genes in $S$ through the estimation of a multi-layer genetic network from gene expression time series and biological knowledge.

The set $S$ chosen is composed of genes that participate of a given biological function. An $n$-layer network is estimated sequentially. The estimation of a layer $L_{i}$ consists in ranking, based in some estimated cost function, the genes influenced by some subset of the genes in the previous layer $L_{i-1}$ and choosing a subset of these genes, based on the rank and on their known relation with the phenomena studied. This process is repeated $n-1$ times and $L_{0}=S$. In each step $i$, the biological functions of $L_{i}$ layer genes are recorded from GO [25] or, eventually, from other functional analysis. This procedure permits to investigate the relation of the phenomena associated to the biological function of $S$ and $L$ genes.

\subsection{Time-Course Microarray}

The input data for our study comes from [22] experiment. This is a time-course microarray experiment, that sampled $T-47 D$ cells over 24 hours through Compugen $19 \mathrm{~K}$ human oligonucleotide array. The total are 16 experiments: the 8 first every hour and the 8 reminders every two hours. The whole experiment was repeated in three different conditions: 1) treated with estrogen (17 $\beta$-estradiol (E2)); 2) treated with estrogen (E2) plus ICI (anti-estrogen component); 3) treated with estrogen (E2) plus 
ICI plus CHX-Cycloheximide (protein synthesis inhibitor component). Each experiment was compared with the $T-47 D$ cells not treated with estrogen. The experiment obtained: 386 genes, estrogen responsive; 139 genes, estrogen responsive and ICI sensitive; 89 genes, estrogen responsive, $I C I$ sensitive and $C H X$ insensitive. These genes were identified as estrogen directly regulated genes.

\subsection{Normalization, Quantization and Filtering}

Let $M$ be the time-course microarray matrix, with $n$ genes in $m$ instants of time. In order to find the best predictors subset of a gene, the pipeline (Figure 1) needs to compute for each subset a cost function associated to it. This process requires that the expression values be discrete values instead of real numbers contained in the output of the microarray experiment. Of several methods to do that, we used a method based on [26]. It consists in two steps:

Normalization of matrix $M$ into the matrix $M_{N}$. It consists in normalizing each gene signal to a signal with normal distribution with expectation equals to 0 and standard deviation equals to 1 . After that, all the genes will have the same distribution and their expressions can be compared. The normalization consists in calculating the expectation $E_{i}$ and the standard deviation $\sigma_{i}$ of the signal for each gene $\mathrm{G}$ given. The resulting elements of the normalized matrix $M_{N}$ are given by

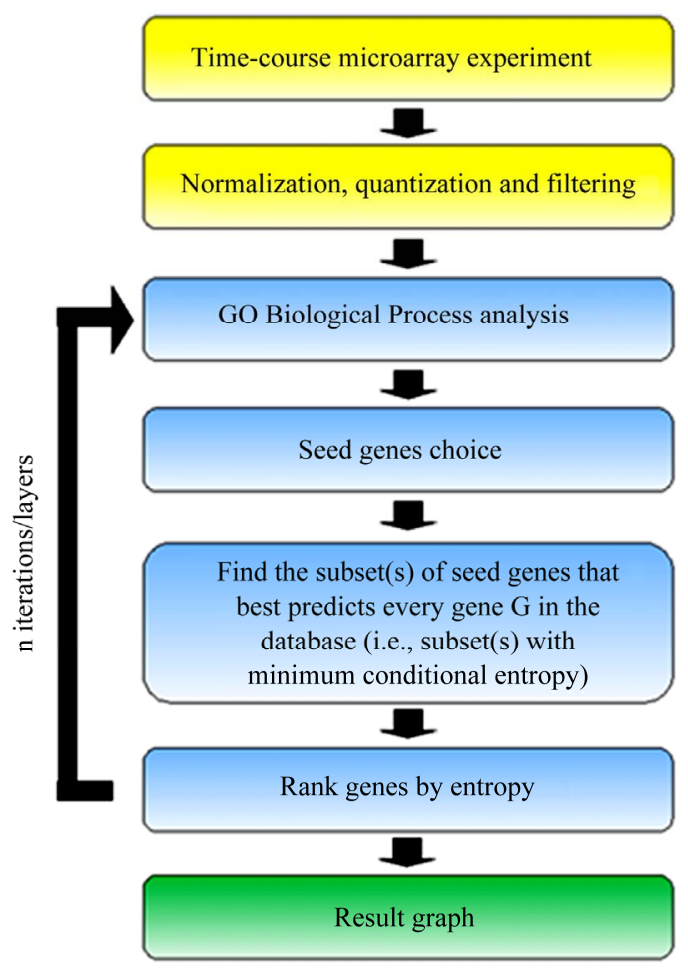

Figure 1. The pipeline steps.

$$
M_{N}[i, k]=\frac{M[i, k]-E_{i}}{\sigma_{i}}
$$

$i=1, \cdots, n$ and $k=1, \cdots, m$.

Quantization of matrix $M_{N}$ into the matrix $M_{Q}$. This process is equivalent to map the normalized signal values in previous step to some qualitative expression levels. In this work, we use three qualitative expression levels: -1 , indicating that the gene is under expressed, 0 , indicating that the gene is null, and 1, indicating that the gene is over expressed in relation to the reference. A threshold mapping is used to perform the quantization as in [26]. For each gene $\mathrm{G}$ a lower $l_{i}$ and an upper $u_{i}$ thresholds are obtained by

$$
l_{i}=\frac{\sum_{M_{N}[i, k]<0} M_{N}[i, k]}{\left|\left\{M_{N}[i, k]: M_{N}[i, k]<0\right\}\right|}
$$

and

$$
u_{i}=\frac{\sum_{M_{N}[i, k]>0} M_{N}[i, k]}{\left|\left\{M_{N}[i, k]: M_{N}>0\right\}\right|}
$$

In other words, $l_{i}$ and $u_{i}$ are the expectation of, respectively, the negative and positive signals. The elements of the quantized matrix $M_{Q}$ are given by:

$$
M_{Q}[i, k]=\left\{\begin{array}{l}
-1, \text { if } M_{N}[i, k]<l_{i} \\
0, \text { if } l_{i} \leq M_{N}[i, k] \leq u_{i} \\
+1, \text { if } M_{N}[i, k]>u_{i}
\end{array}\right.
$$

for $i=1, \cdots, n$ and $k=1, \cdots, m$.

Filtering. In order to avoid further errors, the entries in a time-course microarray experiment must be filtered. To do so, we have to analyze two cases: 1) the expression signal cannot be determined and 2) the gene expression during the experiment is constant. For the first case, we simply set this entry with the null value. In both cases, the genes do not give any new information for the resulted network and for this reason they are removed from the data set.

\subsection{Seed Genes Analysis}

The algorithm pipeline requires at each step a set of genes called seed genes. This set contains the predictors to be found for each gene in the whole data set.

For cells treated with estrogen, for example, in order to test estrogen regulation network, good candidate for seed genes can be genes directly regulated by estrogen, i.e. genes in which estrogen could act as a transcription factor. It is possible to use many tools for analyzing genes biological functions. In this work, we used FunNet [27] software, which calculates the significance P-value of the gene enrichment, of the considered GO/KEGG category, 
with a unilateral Fisher exact test. The genes best predicted by each current set of seed genes are used as predictors for the next step.

\subsection{Cost Function}

For each gene in the whole data set and a given set of seed genes, we try to find the subset of the seed genes that best predict the expression of this gene, which we call target gene.

It is possible to use our method inferring the prediction interactions using several manners (for example, Bayesian networks, ordinary differential equations or other information-theoretic approaches, shown in [6,7].

However, in our tests, we have used the mean conditional entropy as cost function. This measure, which varies between 0 and 1, indicates the dispersion of a probability distribution function, i.e. the entropy has small values for distributions with mass concentration in one of the possible instances and the biggest value for a uniform distribution. For our case, we are interested in the distribution of the expression $G$ of a gene given the vector expression $\boldsymbol{A}$ of a subset of seed genes. Let $Q$ be the set of the discrete values used to quantize the gene expressions (e.g. $Q=\{-1,0,+1\}$ ). And also let $a \in Q^{|A|}$ be an instance of $\boldsymbol{A}$ and $g \in Q$ be an instance of $G$. The mean conditional entropy $E(H(G \mid \boldsymbol{A}))$ is given by:

$$
\begin{aligned}
& E[H(G \mid \boldsymbol{A})]=\sum_{a \in Q^{|| \mid}} p(\boldsymbol{A}=\boldsymbol{a})\left(-\sum_{g \in Q} p(G=g \mid \boldsymbol{A}\right. \\
&\left.=\boldsymbol{a}) \log _{|Q|} p(G=g \mid \boldsymbol{A}=\boldsymbol{a})\right)
\end{aligned}
$$

$$
\hat{p}(\boldsymbol{A}=\boldsymbol{a})= \begin{cases}\frac{N^{+}}{N^{+}+N^{-}} \times \frac{\#(\boldsymbol{A}=\boldsymbol{a})}{N^{+}}, & \text {if } \#(\boldsymbol{A}=\boldsymbol{a}) \geq f ; \\ \frac{N^{-}}{N^{+}+N^{-}} \times \frac{1}{\left|\left\{\boldsymbol{a}^{\prime} \in Q^{|A|}: \#\left(\boldsymbol{A}=\boldsymbol{a}^{\prime}\right)<f\right\}\right|^{\prime}} & \text { if } \#(\boldsymbol{A}=\boldsymbol{a})<f .\end{cases}
$$

The estimator $\hat{p}(\boldsymbol{A}=\boldsymbol{a})$ distributes uniformly the frequency $N$ through the instances that do not occur or occur less than $f$ times.

\subsection{Ranking Results}

We say that a gene $G$ is best predicted than a gene $G$ ' by the seed genes, if the cost of the best predictor subset of the seed genes to $G$ is lower than the cost of the best predictor subset to $G$ '. Ranking the genes of the data set by the costs associated to their best predictor subsets produces a list in which the initial elements are the genes best predicted by the seed genes. This procedure is the key to obtain a new set of genes to the next step of the pipeline. Some methods can be used to choose the next
Based just in the input data one can only calculate an estimation of the cost function in place of its real value. The estimated mean conditional entropy $\hat{E}(H(G \mid \boldsymbol{A}))$ is given by:

$$
\begin{aligned}
& \hat{E}[H(G \mid \boldsymbol{A})]=\sum_{a \in Q^{|A|}} \hat{p}(\boldsymbol{A}=\boldsymbol{a}) \\
& \left(-\sum_{g \in Q} \hat{p}(G=g \mid \boldsymbol{A}=\boldsymbol{a}) \log _{|Q|} \hat{p}(G=g \mid \boldsymbol{A}=\boldsymbol{a})\right)
\end{aligned}
$$

The Equation (2) requires estimation of $P(\boldsymbol{A}=\boldsymbol{a})$ and $P(G=g \mid \boldsymbol{A}=\boldsymbol{a})$. Let $f$ be a frequency threshold used to separate instances $\boldsymbol{a}$ of $\boldsymbol{A}$ that do not occur frequently. Let $N^{+}$be the sum of the frequencies of each instance $\boldsymbol{a}$ of $\boldsymbol{A}$ which occurs more or equal than $f$ and $N$ be the sum of the frequencies of each instance $\boldsymbol{a}$ of $\boldsymbol{A}$ which occurs less than $f$. It is equivalent to:

$$
\begin{aligned}
& N^{+}=\sum_{\#(\boldsymbol{A}=\boldsymbol{a}) \geq f, \forall a \in Q^{|A|}} \#(\boldsymbol{A}=\boldsymbol{a}) . \\
& N^{-}=\sum_{\#(\boldsymbol{A}=\boldsymbol{a}) \geq f, \forall a \in Q^{|A|}} \#(\boldsymbol{A}=\boldsymbol{a})
\end{aligned}
$$

So, the estimator $\hat{p}(G=g \mid \boldsymbol{A}=\boldsymbol{a})$ of $p(G=g \mid \boldsymbol{A}=\boldsymbol{a})$ is given by:

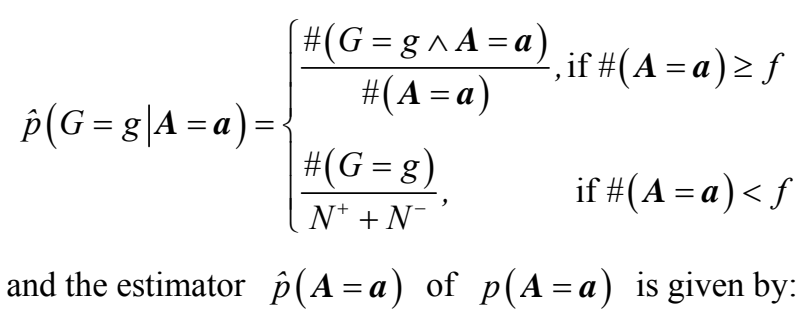

seed genes set. We present here some of them:

Defining a threshold value $e$ to the cost function and extracting only the genes with predictor subset cost lower than $e$. This value can be updated each iteration, depending on the number of genes extracted by this value.

Extracting a fixed number of genes from the top of the ranking list that share some biological function (GO and/or KEGG entries). For example, extract the first 30 genes that have one or more of these biological functions: cell division, cell proliferation and cell cycle.

Simply extracting a fixed number of genes from the top of the ranking list, which can be defined as a percentage of the whole set.

These methods can be grouped to obtain the next seed genes. For example, set a threshold value e and some 
biological functions to extract the genes sharing these biological functions and with best predictor subset cost lower than $e$.

\subsection{Implementation}

P The pipeline was composed by the following steps:

Each iteration was processed by an algorithm using Python that received the seed genes. For each gene $G$ in the set of seed genes, the algorithm executes the following processes:

sub-matrix extraction containing the expressions of the seed genes and the target gene $\mathrm{G}$;

execution of the algorithm to find the best predictor subset;

results storage in an HTML page and in a text file as the source to create the graph on Cytoscape software. This HTML page has, for each gene, a link to Stanford Microarray Database relating the gene to its aliases and known information about it.

The FunNet [27] website (http://www.funnet.info) was used to get the GO Biological Process most enriched categories.

The Cytoscape software (http://www.cytoscape.org) was used to build the network graph image.

The experimental results were processed in an Dual Core AMD Opteron ${ }^{\mathrm{TM}}$ with $4 \mathrm{~GB}$ of RAM. The resulting graph, the seed genes for each iteration and the source code can be downloaded at http://bioinfo.lbhc.hcancer.org.br/pipeline/.

\section{Results}

We performed two experiments based on the same data. The input for the experimental results was a time-course microarray (Compugen 19K human oligonucleotide array) experiment of $T-47 D$ cells treated with estrogen (E2) [22] in 16 experiments over 24 hours.

Some genes were removed from the whole process: 1) the genes not found in GO Biological Process; 2) genes with constant signal expression and 3) genes with more than half of the signal expression undefined. For the undefined signals the entries were marked with null values and ignored during the whole process. The GO entry was assigned to each gene, the complete data were normalized and quantized according to the methods discussed previously and we used 3 levels of quantization: $-1,0$ and +1 . We used $f=1$ as the frequency threshold explained in Section 2.5. In our experiments, we chose a general maximum threshold value of 0.15 , because we want to be restrictive in relation to the cost function. Another general restriction is that in each iteration the method permits to adjoin to the network less than $5 \%$ of the whole set of candidate genes. In order to do that, the threshold of 0.15 may be decreased.

The initial seed genes were obtained from the 386 genes E2-responsive in [22]. As we are interested in the role of estrogen in proliferation of cancer cells, in our first experiment, from these 386 genes, we searched for the ones with GO Biological Process related to "Cell proliferation" and found 30 genes (level 9, P-value of 0.00314).

This biological function used to filter the initial seed genes was obtained from the work in [19] as one of the functional categories of genes stimulated or inhibited by estrogen. In Figure 2 we see the whole network of this experiment. By using the 30 "Cell proliferation" related seed genes, we grew the network over 3 layers, of which the first one corresponds to the seed genes. In order to find the second layer we added to the network the genes that were predicted by the seed genes with mean conditional entropy lower than 0.15 , resulting in a layer with 572 genes, from which 105 have GO Biological Process annotation. Then, from these 572, we performed a search for the genes also related to "Cell proliferation" (in $G O$ Biological Process), finding 8 genes (level 6, P-value of 0.0299). After that, these 8 genes were also used as seed genes to grow the third layer. The third layer is composed by the genes predicted with mean conditional entropy lower than 0.15 , a set of 28 genes. We searched in the literature for these genes that were related to cancer, and found 18 of them, as shown in Supplementary Table $1^{1}$.

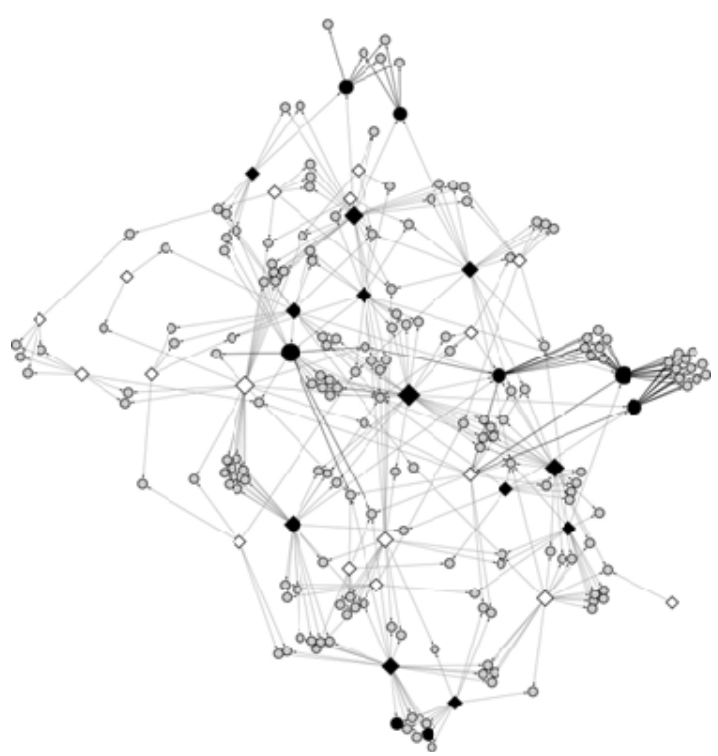

Figure 2. The "Cell proliferation" 3 layers network. The diamond nodes are the seed genes. The black nodes are the second layer genes related to "Cell proliferation" in GO Biological Process or their predictors. The white genes are the seed genes that are not predictors of genes related to "Cell proliferation", that are the gray ones. The darker arrows indicate the predictions from second to third layer genes. The size of the nodes are proportional to the number of linkages.

${ }^{1}$ See: http://bioinfo.lbhc.hcancer.org.br/pipeline/. 
In the group of 386 genes that [22] discovered as influenced by estrogen, they performed an experiment using chromatin immunoprecipitation (ChIP) to characterize the interaction between ER and the regulatory elements of candidate target genes. Through this experiment they found 89 genes. As we previously explained, it is our interest to discover what and how genes directly regulated by estrogen are related to each other and to other genes. Therefore, we used these 89 genes as seed genes in our second experiment. We performed the search of all of them in GO Biological Process in order to find the annotated ones. This search resulted in a group of 53 genes, which were used as the seed genes. Then, we added to the network (Figure 3), which has only two layers, the genes that were predicted by the seed genes with mean conditional entropy lower than 0.08 (as described previously, this threshold adds to the network less than $5 \%$ of the whole set of genes). It resulted in a layer with 412 genes.

After that, we performed a search among the biological functions of these 412 genes in Gene Ontology. Several biological functions known to be related to estrogen (like "Cell proliferation" and "Cell differentiation") were found. In some levels of the GO classification (Figure 4), we discovered a function related to estrogen that is not so prominent in the literature. Out of the 412 genes predicted by the 53 initial seed genes, 27 are related to "Cell adhesion". In the first levels of GO enrichment analysis, "Cell adhesion" appeared as the most enriched biological function category. This is an important discovery, because it is known that cell adhesion has a direct relationship to cancer morphogenesis [28]. Roughly speaking,

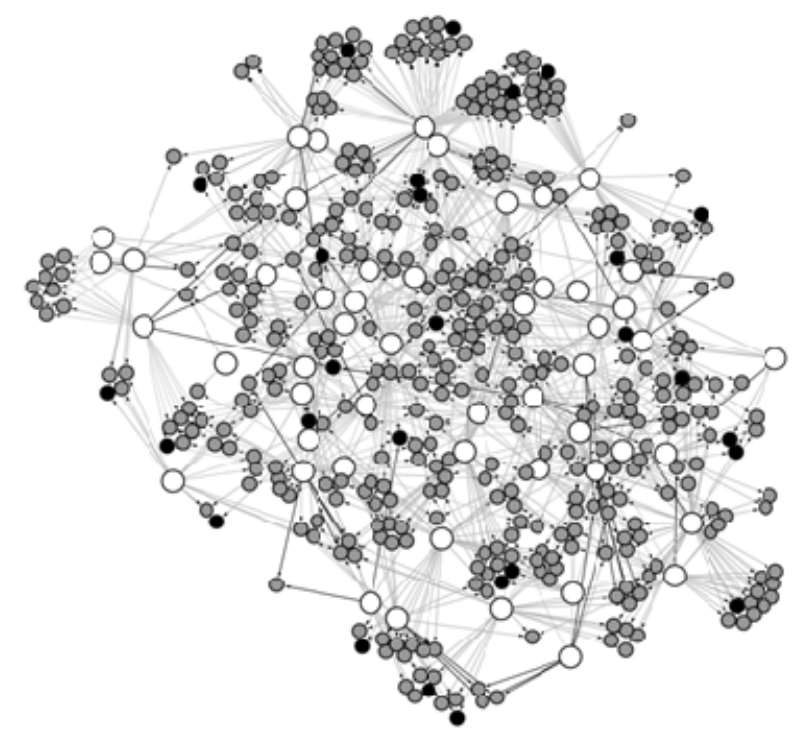

Figure 3. "Cell adhesion" network. The white nodes are the seed genes. The gray ones are the genes predicted by the seed genes and the black ones are the predicted genes related to “Cell adhesion” in GO Biological Process.

\section{GO Biological Process}

Set: 412 genes

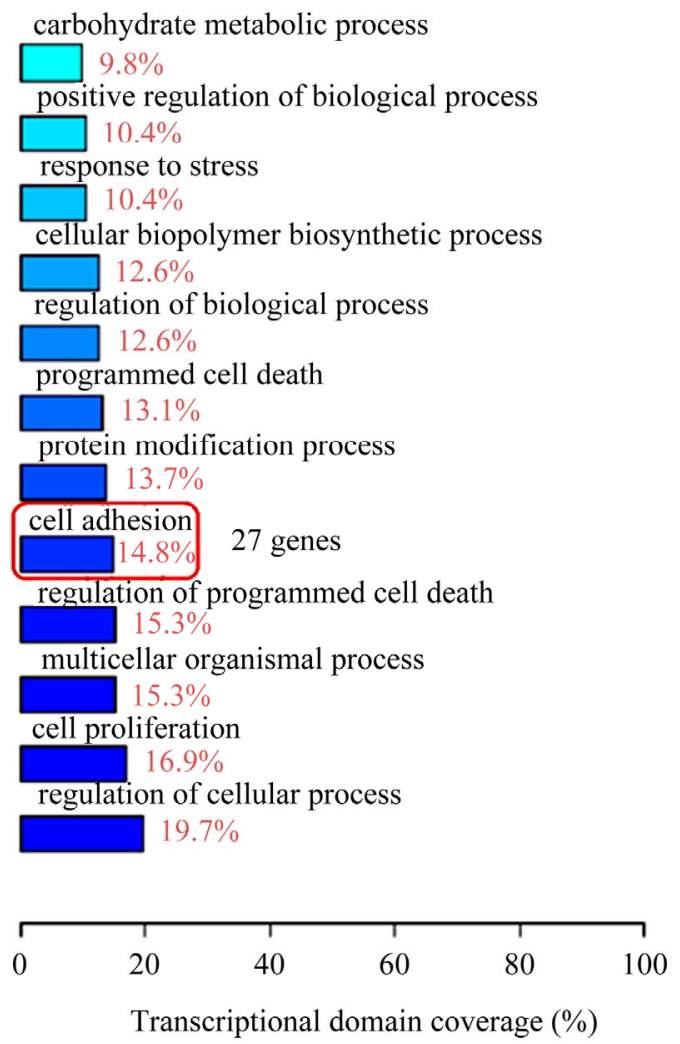

Figure 4. List of 12 most enriched "GO Biological Process" categories (level 8), in which "Cell adhesion" is present. Of the 412 genes predicted by the first layer, 27 of the annotated ones (183) are related to "Cell adhesion".

reduced intercellular adhesiveness allows cancer cells to disobey the social order, resulting in destruction of histological structure, which is the morphological hallmark of malignant tumors.

\section{Discussion}

Our pipeline is suitable for small data sets, takes into account the biological knowledge and contributed for understanding the physiopathology of breast cancer induced by estrogen. It also has not high system requirements (for example, the user can easily run the program in a $256 \mathrm{MB}$ of system memory, a $2 \mathrm{GHz}$ processor and 1 GB of disk space). Using the predicted genes by seeds related to cell proliferation we can propose new genes involved in the tumor proliferation. It is important to note that from 28 genes proposed as important genes related to tumor proliferation in breast cancer 18 have been validated in the literature as cancer proliferating genes. Another important information to cite is that in the first experiment (Figure 5) we can observe that the second 

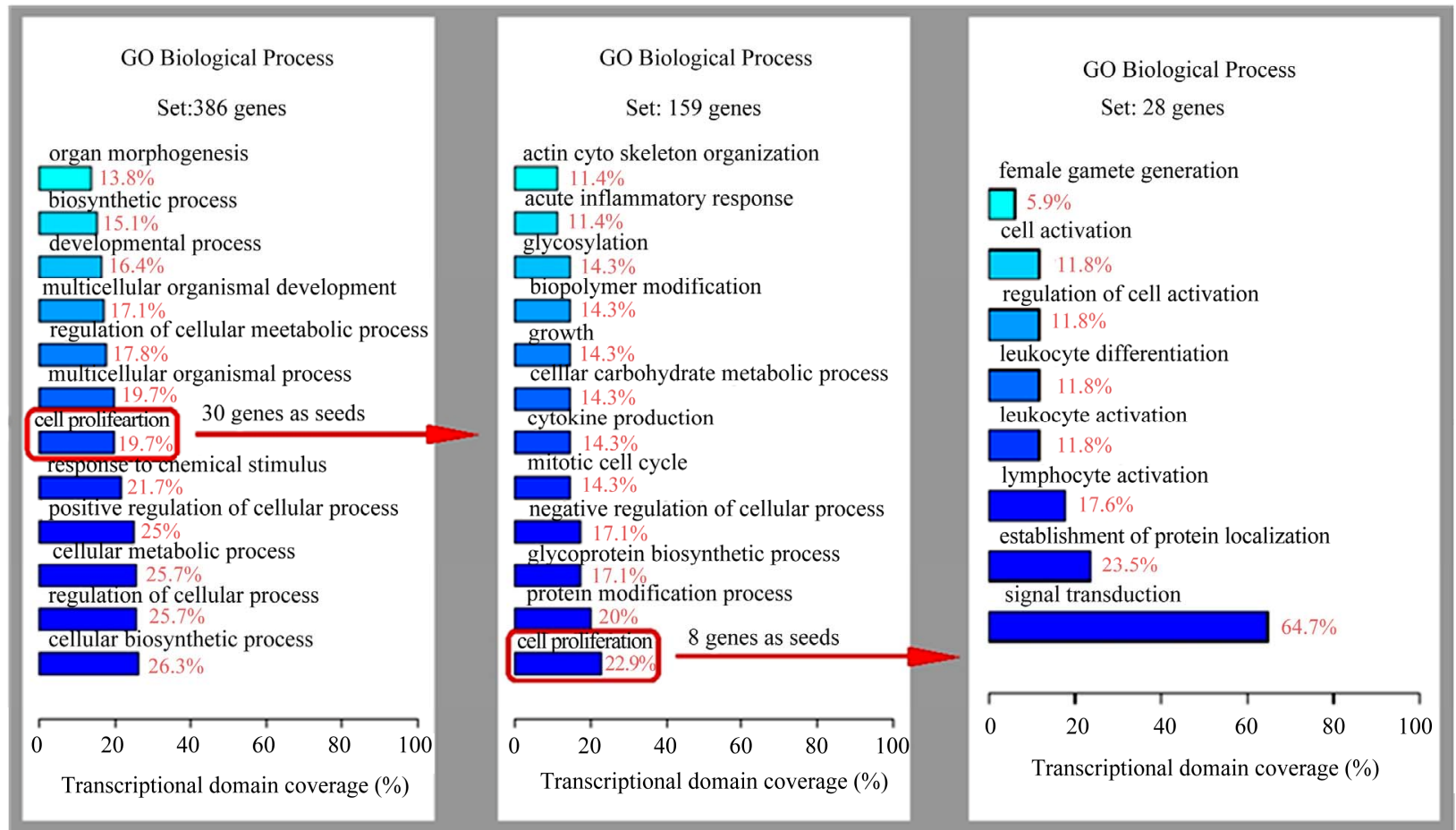

Figure 5. The GO Biological Process 12 most enriched categories by the genes of our first experiment. Each column corresponds to one layer. In the first and in the second layer we chose only the genes related to "Cell proliferation" to be the predictor genes of the next step. Figure generated by Funnet [27].

layer "Cell proliferation" related genes predictors are the ones with more linkages (the hub ones). It confirms the importance that these genes have in the network [29].

The metastasis process consists in a complex sequence of events involving the tumor cells and properties of the host organism [30]. The detachment of the tumor cells of the primary tumor is considered the first and more important event in the metastatic process. The tumor cells can be easier separated from a compact tumor tissue than normal cells near a normal tissue [31]. This separation of tumor cells is regulated by the cell adhesion property of the tumor. The cell adhesion biological function is applied to genes related to adhesion molecules, those acting as positive or negative modulators in the metastasis process $[32,33]$. Despite the rapid progress in the understanding of cell adhesion biology, the few available data turns hard the proposition of a simple model in which the cell adhesion molecules can be related to the tumor growing and metastasis. Studies where tumor cells are injected intravenously have, in general, shown an increase in the adhesion function of these cells and had a positive correlation to the metastatic ability. Those studies have a bias in determining that the high adhesion property of these cells makes that they have more facility to bind to circulation cells and be deposited in different regions of the organism. On the other hand, studies that implant tumor tissues in organisms allowing them to grow and promote spontaneous metastasis have, in general, demonstrated an inverse relation to cell adhesion function and metastatic ability. The relation of estrogen as regulator of genes related to cell adhesion is not very prominent in the literature. In this work, we could obtain, from a time-course microarray experiment using cells submitted to estrogen, a strong evidence of estrogen regulating genes related to cell adhesion. From an initial list of 53 genes directly regulated (also called direct targets) by estrogen [22], we obtained a list with 27 genes related to cell adhesion. For each one of these 27 genes, a prediction table, relating the gene to the initial list, was constructed.

These results indicate a strong relation between estrogen and cell adhesion genes, which could have a role in metastasis. The most of those genes have been related to invasion and metastatic process in cancer as can be seen in Supplementary Table $2^{2}$. Five have already been associated with breast cancer validating our approach to search for genes related to breast cancer proliferationinduced by estrogen: BCAR3 regulates Src/p130 Cas asso ciation, Src kinase activity, and breast cancer adhesion ropilin-2 expression in breast cancer correlates with ly$\mathrm{mph}$ node metastasis and poor prognosis. Tumour-associated tenascin-C isoforms promote breast cancer cell in-

\footnotetext{
${ }^{2}$ See: http://bioinfo.lbhc.hcancer.org.br/pipeline/.
} 
vasion and growth by matrix metalloproteinase-dependent and independent mechanisms. SPOCK (SPARC) is a proteoglycan reported to be associated to poor outcome in breast cancer [34] and resistance to first-line tamoxifen treatment [35]. It is also interesting to note that some genes related to families of proteins implicated in the developing nervous system may play an important role in cancer [36,37] and we found some of them: PTPRS, CNTNAP2, NRP2, LSAMP, KITL6, NEO1 and L1CAM.

\section{Acknowledgements}

The authors are grateful to FAPESP (99/12765-2, 01/094 01-0, 04/03967-0 and 05/00587-5), CNPq (300722/98-2, 468 413/00-6, 521097/01-0 474596/04-4 and 491323/ 05-0) and CAPES for financial support. This work was partially supported by grant 1 D43 TW07015-01 from the National Institutes of Health, USA.

\section{REFERENCES}

[1] M. Schena, "DNA Microarrays: A Practical Approach," Oxford University Press, Oxford, 1999.

[2] S. Marguerat and J. Bähler, "Rna-Seq: From Technology to Biology," Cellular and Molecular Life Sciences, Vol. 67, No. 4, 2010, pp. 569-579. doi:10.1007/s00018-009-0180-6

[3] M. Schena, D. Shalon, R. Davis and P. O. Brown, "Quantitative Monitoring of Gene Expression Patterns with a Complementary DNA Microarray," Science, Vol. 270, No. 5235,1995 , pp. 467-470. doi:10.1126/science.270.5235.467

[4] S. Peddada, E. Lobenhofer, L. Li, C. Afshari, C. Weinberg and D. M. Umbach, "Gene Selection and Clustering for Time-Course and Dose-Response Microarray Experiments Using Order-Restricted Inference," Science, Vol. 19, No. 7, 2003, pp. 834-841.

[5] R. F. Hashimoto, S. Kim, I. Shmulevich, W. Zhang, M. L. Bittner and E. R. Dougherty, "Growing Genetic Regulatory Networks from Seed Genes," Bioinformatics, Vol. 20, No. 8, 2004, pp. 1241-1247. doi:10.1093/bioinformatics/bth074

[6] M. Bansal, V. Belcastro, A. Ambesi-Impiombato and D. di Bernardo, "How to Infer Gene Networks from Expression Profiles," Molecular Systems Biology, Vol. 3, No. 78, 2007, pp. 1-10.

[7] H. D. Jong, "Modeling and Simulation of Genetic Regulatory Systems: A Literature Review," Journal of Computational Biology, Vol. 9, No. 1, 2002, pp. 67-103. doi: $10.1089 / 10665270252833208$

[8] I. Shmulevich and E. R. Dougherty, "Genomic Signal Processing," Princeton University Press, Princeton, 2007.

[9] C. Sima, J. Hua and S. Jung, "Inference of Gene Regulatory Networks Using Time-Series Data: A Survey," Current Genomic, Vol. 10, No. 6, 2009, pp. 416-429. doi: $10.2174 / 138920209789177610$
[10] E. Segal, M. Shapira, A. Regev, D. Pe'er, D. Botstein, D. Koller and N. Friedman, "Module Networks: Identifying Regulatory Modules and Their Condition-Specific Regulators from Gene Expression Data," Nature Genetics, Vol. 34, No. 2, 2003, pp. 166-176. doi:10.1038/ng1165

[11] S. A. Kauffman, "The Origins of Order, Self-Organization and Selection in Evolution," Oxford University Press, Oxford, 1993, pp. 441-520.

[12] I. Shmulevich, E. R. Dougherty, S. Kim and W. Zhang, "Probabilistic Boolean Networks: A Rule-Based Uncertainty," Bioinformatics, Vol. 18, No. 2, 2002, pp. 261274. doi:10.1093/bioinformatics/18.2.261

[13] J. Barrera, R. M. Cesar-Jr., D. C. Martins-Jr., E. F. Merino, R. Z. N. Vêncio, F. G. Leonardi, M. M. Yamamoto, C. A. B. Pereira and H. A. Portillo, "A New Annotation Tool for Malaria Based on Inference of Probabilistic Genetic Networks," In CAMDA, 2004.

[14] P J. M. eña, J. Björkegren and J. Tegnér, "Growing Bayesian Network Models of Gene Networks from Seed Genes," Bioinformatics, Vol. 21, Suppl. 2, 2005, pp. ii224-ii229. doi:10.1093/bioinformatics/bti1137

[15] X. Xu, L. Wang and D. Ding, "Learning Module Networks from Genome-Wide Location and Expression Data," FEBS Letters, Vol. 578, No. 3, 2004, pp. 297-304. doi:10.1016/i.febslet.2004.11.019

[16] B. T. Zhu and A. H. Conney, "Functional Role of Estrogen Metabolism in Target Cells: Review and Perspectives," Carcinogenesis, Vol. 19, No. 1, 1998, pp. 1-27. doi:10.1093/carcin/19.1.1

[17] C. Förster, S. Mäkela, A. Wärri, S. Kietz, D. Becker, K. Hultenby, M. Warner and J. Gustafsson, "Involvement of Estrogen Receptor $\beta$ in Terminal Differentiation of Mammary Gland Epithelium," Proceedings of the $\mathrm{Na}$ tional Academy of Sciences, Vol. 99, No. 24, 2002, pp. 15578- 15583. doi:10.1073/pnas.192561299

[18] K. R. Coser, J. Chesnes, J. Hur, S. Ray, K. J. Isselbacher and T. Shioda, "Global Analysis of Ligand Sensitivity of Estrogen Inducible and Suppressible Genes in mcf7/Bus Breast Cancer Cells by DNA Microarray," Proceedings of the National Academy of Sciences, Vol. 100, No. 24, 2003, pp. 13994-13999. doi:10.1073/pnas.2235866100

[19] J. Frasor, J. M. Danes, B. Komm, K. C. N. Chang, C. R. Lyttle and B. S. Katzenellenbogen, "Profiling of Estrogen Up- and Down-Regulated Gene Expression in Human Breast Cancer Cells: Insights into Gene Networks and Pathways Underlying Estrogenic Control of Proliferation and Cell Phenotype," Endocrinology, Vol. 144, No. 10, 2003, pp. 4562-4574. doi:10.1210/en.2003-0567

[20] V. X. Jin, Y. W. Leu, S. Liyanarachchi, H. Sun, M. Fan, K. P. Nephew, T. H. Huang and R. V. Davuluri, "Identifying Estrogen Receptor $\alpha$ Target Genes Using Integrated Computational Genomics and Chromatin Immunoprecipitation Microarray," Nucleic Acids Research, Vol. 32, No. 22, 2004, pp. 6627-6635. doi:10.1093/nar/gkh1005

[21] A. S. Levenson, I. L. Kliakhandler, K. M. Svoboda, K. M. Pease, S. A. Kaiser, J. E. Ward-III and V. C. Jordan, "Molecular Classification of Selective Oestrogen Receptor Modulators on the Basis of Gene Expression Profiles of Breast Cancer Cells Expressing Oestrogen Receptor 
$\alpha$," British Journal of Cancer, Vol. 87, No. 4, 2002, pp. 449-456. doi:10.1038/sj.bjc. 6600477

[22] C. Lin, A. Ström, V. B. Vega, S. L. Kong, A. L. Yeo, J. S. Thomsen, W. C. Chan, B. Doray, D. K. Bangarusamy, A. Ramasamy, L. A. Vergara, S. Tang, A. Chong, V. B. Bajic, L. D. Miller, J. Gustafsson and E. T. Liu, "Discovery of Estrogen Receptor $\alpha$ Target Genes and Response Elements in Breast Tumor Cells," Genome Biology, Vol. 5, No. 9, 2004, pp. 1-18. doi:10.1186/gb-2004-5-9-r66

[23] A. Weisz, W. Basile, C. Scafoglio, L. Altucci, F. Bresciani, A. Facchiano, P. Sismondi, L. Cicatiello and M. Bortoli, "Molecular Identification of ER $\alpha$-Positive Breast Cancer Cells by the Expression Profile of an Intrinsic Set of Estrogen Regulated Genes," Journal Cellular Physiology, Vol. 200, No. 3, 2004, pp. 440-450. doi:10.1002/jep.20039

[24] M. L. Whitfield, L. K. George, G. D. Grant and C. M. Perou, "Common Markers of Proliferation," Nature Reviews, Vol. 6, No. 2, 2006, pp. 99-106. doi: $10.1038 / \mathrm{nrc1} 1802$

[25] M. Ashburner, C. A. Ball, J. A. Blake, D. Botstein, H. Butler, J. M. Cherry, A. P.Davis, K. Dolinski, S. S. Dwight, J. T. Eppig, M. A. Harris, D. P. Hill, L. Issel-Tarver, A. Kasarskis, S. Lewis, J. C. Matese, J. E. Richardson, M. Ringwald, G. M. Rubin and G. Sherlock,. "Gene Ontology: Tool for the Unification of Biology," Nature Genetics, Vol. 25, 2000, pp. 25-29. doi:10.1038/75556

[26] J. Barrera, R. M. Cesar-Jr, D. C. Martins-Jr., R. Z. N. Vêncio, E. F. Merino, M. M. Yamamoto, F. G. Leonardi, C. A. B. Pereira and H. A. del Portillo, "Constructing Probabilistic Genetic Networks of Plasmodium Falciparum from Dynamical Expression Signals of the Intraerythrocytic Development Cycle, Chapter 2," Springer, Berlin, 2006, pp. 11-26.

[27] E. Prifti, J.-D. Zucker, K. Clement and C. Henegar, "Funnet: An Integrative Tool for Exploring Transcriptional Interactions," Bioinformatics, Vol. 24, No. 22, 2008, pp. 2636-2638. doi:10.1093/bioinformatics/btn492

[28] S. Hirohashi and Y. Kanai, "Cell Adhesion System and Human Cancer Morphogenesis," Cancer Science, Vol. 94, No. 7, 2003, pp. 575-581. doi:10.1111/j.1349-7006.2003.tb01485.x

[29] H. Jeong, S. P. Mason, A.-L. Barabasi and Z. N. Oltvai, "Lethality and Centrality in Protein Networks," Nature,
Vol. 411, No. 6833, 2001, pp. 41-42. doi: $10.1038 / 35075138$

[30] J. I. Fidler, "Origin and Biology of Cancer Metastasis," Cytometry, Vol. 10, No. 6, 1989, pp. 673-680. doi:10.1002/cyto. 990100602

[31] D. R. Coman, "Adhesiveness and Stickiness: Two Independent Properties of the Cell Surface," Cancer Research, Vol. 1, 1961, pp. 1436-1438.

[32] R. O. Hynes, "Integrins: Versatility, Modulation, and Signaling in Cell Adhesion," Cell, Vol. 69, No. 1, 1992, pp. 11-25. doi:10.1016/0092-8674(92)90115-S

[33] H. Oka, H. Shiozaki, K. Kobayashi, M. Inoue, H. Tahara, T. Kobayashi, Y. Takatsuka, N. Matsuyoshi, S. Mirano, M. Takeichi and T. Mori, "Expression of E-Cadherin Cell Adhesion Molecules in Human Breast Cancer Tissues and Its Relationship to Metastasis," Cancer Research, Vol. 53, No. 7, 1993, pp.1696-1701.

[34] J. Helleman, M. P. Jansen, K. Ruigrok-Ritstier, I. L. van Staveren, M. P. Look, M. E. M. van Gelder, A. M. Sieuwerts, J. G. Klijn, S. Sleijfer, F J. A. oekens and E. M. Berns, "Association of an Extracellular Matrix Gene Cluster with Breast Cancer Prognosis and Endocrine Therapy Response," Clinical Cancer Research, Vol. 14, No. 17, 2008, pp. 5555-5564. doi:10.1158/1078-0432.CCR-08-0555

[35] M. P. H. M. Jansen, K. Ruigrok-Ritstier, L. C. J. Dorssers, I. L. van Staveren, M. P. Look, M. E. M. van Gelder, A. M. Sieuwerts, J. Helleman, S. Sleijfer, J. G. M. Klijn, J. A. Foekens and E. M. J. J. Berns, "Down Regulation of Siah2, an Ubiquitin e3 Ligase, Is Associated with Resistance to Endocrine Therapy in Breast Cancer," Breast Cancer Research and Treatment, Vol. 116, No. 2, 2009, pp. 263-271. doi:10.1007/s10549-008-0125-Z

[36] G. E. Ayala, H. Dai, M. Powell, R. Li, Y. Ding, T. M. Wheeler, D. Shine, D. Kadmon, T. Thompson, B. J. Miles, M. M. Ittmann and D. Rowley, "Cancer-Related Axonogenesis and Neurogenesis in Prostate Cancer," Clinical Cancer Research, Vol. 14, No. 23, 2008, pp. 7593-7603. doi:10.1158/1078-0432.CCR-08-1164

[37] A. Chédotal, G. Kerjan and C. Moreau-Fauvarque, "The Brain within the Tumor: New Roles for Axon Guidance Molecules in Cancers," Cell Death Differ, Vol. 12, No. 8, 2005, pp. 1044-1056. doi:10.1038/sj.cdd.4401707 